\title{
PARKINSONISMO INDUZIDO PELA FLUNARIZINA
}

\author{
PAULO N. DISCHER DE SA* - LIANA MIRIAM MIRANDA HEINISCH ***
}

RESUMO - Os autores estudaram 19 pacientes com parkinsonismo induzido pela flunarizina. A retirada da medicaçāo levou ao desaparecimento dos sinais e sintomas em todos os casos em periodo de tempo que variou de 7 dias a 10 meses. Depressão foi observada em $68,5 \%$ da amostra.

\section{Parkinsonism induced by flunarizine.}

SUMMARY - The authors studied 19 patients with parkinsonism induced by flunarizine. All them improved when the drug therapy was discontinued for periods from 7 days to 10 months. Depression was observed in $68.5 \%$ of the patients.

Flunarizina $(\mathrm{Fz})$, um derivado difluorinado da cinarizina $(\mathrm{Cz})$, é bloqueador seletivo do canal de cálcio, com atividade anti-histamínica, anti-serotoninérgica e anti-dopaminérgica, apresentando meia-vida plasmática longa 2,4,5,8. Fz é 2,5 a 15 vezes mais potente que a $\mathrm{Cz}_{5}$ e tem sido amplamente utilizada para vertigem de origem central ou periférica, distúrbios do fluxo sanguíneo cerebral, doença vascular periférica oclusiva, profilaxia de enxaqueca e, mesmo, para crises convulsivas 4,5. Os efeitos colaterais mais frequentes são sonolência, astenia, cefaléia, epigastralgia, náusea, insônia, depressão e 'rash' cutâneo 4,5. Chouza et al.4 descreveram complicaçōes extrapiramidais induzidas pela $\mathrm{Fz}$ e Micheli et al.8 registraram os movimentos anormais provocados pela $\mathrm{Fz}$ e $\mathrm{Cz}$.

Estudamos 19 pacientes com parkinsonismo induzido pela Fz, com o objetivo de enfatizar os efeitos iatrogênicos extrapiramidais.

\section{CASUISTICA}

No periodo de maio/84 a janeiro/88 foram observados 19 pacientes com sindrome de Parkinson, sendo 2 homens e 17 mulheres, com média de idade de 66 anos (37-76 anos). A dose de flunarizina variou de 10 a $40 \mathrm{mg}$ (media, $16 \mathrm{mg} / \mathrm{d}$ ). Nenhum paciente fazia uso de outras drogas que pudessem ser responsáveis pelo quadro clínico. Depressão foi observada em 13 pacientes $(68,5 \%)$ sendo leve em três, moderada em 9 e severa em um. Não houve sucesso por tratamento realizado com L-dopa e anticolinérgico. A retirada da medicação

Trabalho realizado no Serviço de Neurologia do Hospital Governador Celso Ramos (HGCR), Florianopolis: * Professor Adjunto da Disciplina de Neurologia do Departamento de Clínica Médica da Universidade Federal de Santa Catarina; ** Médica Residente de Neurologia. 
(Fz) levou ao desaparecimento de sinais e sintomas em todos os casos, em período de tempo que variou de 7 dias (caso 12) a 10 meses (caso 16). As características gerais e manifestações clínicas dos 19 pacientes encontram-se nas tabelas 1 e 2 .

\begin{tabular}{|c|c|c|c|c|c|c|c|}
\hline Caso & $\begin{array}{l}\text { Idade } \\
\text { (anos) }\end{array}$ & Sexo & Doença básica & $\begin{array}{l}\text { Dose } \\
(\mathrm{mg})\end{array}$ & $\begin{array}{l}\text { Tempo de } \\
\text { uso/inicio } \\
\text { sintomas }\end{array}$ & Tratamento & Evolução \\
\hline 1 & 75 & M. & Vertigens & 20 & $10 \mathrm{~m}$ & $\begin{array}{l}\text { Anticolinérgico } \\
+ \text { L-Dopa }\end{array}$ & $30 \mathrm{~d}$ \\
\hline 2 & 62 & $F$ & Vertigens & 20 & $2 \mathrm{~m}$ & $\begin{array}{l}\text { Anticolinérgico } \\
+ \text { Prolopa }\end{array}$ & $4 \mathrm{~m}$ \\
\hline 3 & 71 & M & Vertigens & 20 & $9 \mathrm{~m}$ & nada & $30 \mathrm{~d}$ \\
\hline 4 & 76 & $\mathbf{F}$ & Vertigens & 10 & $2 \mathrm{~m}$ & Anticolinérgico & $60 \mathrm{~d}$ \\
\hline 5 & 72 & F & Depressão & 10 & + de 1 a & L-Dopa & $6 \mathrm{~m}$ \\
\hline 6 & 65 & $F$ & Vertigens & 10 & $8 \mathrm{~m}$ & nada & $4 \mathrm{~m}$ \\
\hline 7 & 37 & $F$ & Vertigens & 20 & $8 \mathrm{~m}$ & nada & $4 \mathrm{~m}$ \\
\hline 8 & 67 & $F$ & 'Labirintite' & 10 & $7 \mathrm{~m}$ & Anticolinérgico & $3 \mathrm{~m}$ \\
\hline 9 & 64 & $\mathrm{~F}$ & 'Labirintite' & 10 & $5 \mathrm{~m}$ & $\begin{array}{l}\text { Anticolinérgico } \\
\text { + imipramina }\end{array}$ & $60 \mathrm{~d}$ \\
\hline 10 & 54 & F & Vertigens & 10 & $6 \mathrm{~m}$ & $\begin{array}{l}\text { Anticolinérgico } \\
+ \text { imipramina }\end{array}$ & $3 \mathrm{~m}$ \\
\hline 11 & 69 & $F$ & Vertigens & 10 & $1 \mathrm{a}$ & nada & $50 \mathrm{~d}$ \\
\hline 12 & 47 & $F$ & Vertigens & 10 & $15 \mathrm{~d}$ & nada & $7 \mathrm{~d}$ \\
\hline 13 & 72 & $F$ & Vertigens & 10 & $11 \mathrm{~m}$ & nada & $30 \mathrm{~d}$ \\
\hline 14 & 72 & $F$ & Vertigens & 10 & $?$ & $\begin{array}{l}\text { Imipramina } \\
+ \text { L-Dopa }\end{array}$ & $60 \mathrm{~d}$ \\
\hline 15 & 68 & $F$ & Vertigens & 10 & $?$ & Anticolinérgico & $60 \mathrm{~d}$ \\
\hline 16 & 76 & $F$ & Vertigens & 40 & $11 \mathrm{~m}$ & Anticolinérgico & $10 \mathrm{~m}$ \\
\hline 17 & 74 & $\mathrm{~F}$ & Vertigens & 20 & $5 \mathrm{~m}$ & L-Dopa & $60 \mathrm{~d}$ \\
\hline 18 & $?$ & $F$ & Vertigens & 40 & $7 \mathrm{~d}$ & nada & $20 \mathrm{~d}$ \\
\hline 19 & 66 & $F$ & Vertigens & 40 & $3 \mathrm{~m}$ & Imipramina & $60 d$ \\
\hline
\end{tabular}

Tabela 1 - Características gerais de 19 pacientes com parkinsonismo induzido pela flunarizina; todos eram de raca branca. Evoluçä, tempo de desaparecimento dos sinais $e$ sintomas após retirada da flunarizina; $a$, anos; $m$, meses; $d$, dias.

\section{COMENTARIOS}

O parkinsonismo é a desordem neurológica năo vascular mais comum no idoso. As manifestaçōes extrapiramidais são induzidas por drogas em inúmeras vezes. O parkinsonismo pode ser induzido por neurolépticos, benzodiazepinicos, metoclopramida e metildopa 1,3,6-12. Stephen e Williamson 10 observaram que, de 95 novos casos de parkinsonismo admitidos em departamento de geriatria, $51 \%$ eram induzidos por drogas.

A Fz tem sido usada como vasodilatador e sedativo labiríntico. Apesar de não termos idéia da freqüência das manifestaçōes extrapiramidais, achamos conveniente estarmos atentos para o aparecimento de novos casos, tentando detectar precocemente os efeitos iatrogênicos. 


\begin{tabular}{|c|c|c|c|c|c|c|}
\hline \multirow[b]{2}{*}{ Caso } & \multirow[b]{2}{*}{ Modo de inicio } & \multicolumn{4}{|c|}{ Manifestaçōes clínicas } & \multirow[b]{2}{*}{ Drogas concomitantes } \\
\hline & & Rigidez & Tremor & Acinesia & Depressão & \\
\hline 1 & Tremor & + & +++ & ++ & - & Benzodiazepinico \\
\hline 2 & $\begin{array}{l}\text { Ausência de automa- } \\
\text { tismos + facies } \\
\text { parkinsoniana }\end{array}$ & ++ & +++ & $+t+$ & + & Benzodiazepínico \\
\hline 3 & Tremor mandibular & - & + & + & - & Dipiridamol \\
\hline 4 & $\begin{array}{l}\text { Tremor mandibular } \\
+ \text { mão } \mathrm{D}\end{array}$ & - & $+t+$ & - & - & Benzodiazepínico \\
\hline 5 & $\begin{array}{l}\text { Facies parkinsoniana } \\
+ \text { bradicinesia }\end{array}$ & - & $\leftarrow$ & $+t+$ & +++ & $\begin{array}{l}\text { Benzodiazepínico }+ \\
\text { verapamil }\end{array}$ \\
\hline 6 & Bradicinesia & - & - & $++t$ & - & Hidroclorotiazida \\
\hline 7 & $\begin{array}{l}\text { Bradicinesia } \\
+ \text { depressão }\end{array}$ & - & - & $++t$ & ++ & Benzodiazepínico \\
\hline 8 & Tremor & - & $+t+$ & - & + & $\begin{array}{l}\text { Benzodiazepínico } \\
\text { hidroclorotiazida }\end{array}$ \\
\hline 9 & $\begin{array}{l}\text { Tremor + depressão } \\
+ \text { facies parkinsoniana }\end{array}$ & - & + & + & $++t+$ & Benzodiazepínico \\
\hline 10 & Tremor & $t+$ & $t$ & $+t$ & ++ & $\begin{array}{l}\text { Benzodiazepínico }+ \\
\text { imipramina }\end{array}$ \\
\hline 11 & Depressão & - & $一$ & $+t$ & ++ & Enalapril \\
\hline 12 & Depressão & - & - & ++ & ++ & Benzodiazepínico \\
\hline 13 & Depressāo & - & - & $+t$ & ++ & $\begin{array}{l}\text { Dipiridamol }+ \text { isossor- } \\
\text { bida }+ \text { nifedipina }\end{array}$ \\
\hline 14 & Dificuldade à marcha & +++ & + & ++ & ++ & Metil-dopa + digoxina \\
\hline 15 & Tremor & + & $+t+$ & $+t+$ & + & $\begin{array}{l}\text { Hidroclorotiazida }+ \\
\text { amiodarona }\end{array}$ \\
\hline 16 & Tremor & - & ++ & + & - & $\begin{array}{l}\text { Clorpropamida }+ \\
\text { fenobarbital }\end{array}$ \\
\hline 17 & Dificuldade à marcha & - & - & ++ & - & Clorpropamidia \\
\hline 18 & Tremor + depressão & + & ++ & + & ++ & Quinidina \\
\hline 19 & Tremor + depressão & ++ & ++ & ++ & +++ & $\begin{array}{l}\text { Clorpropamida }+ \text { digo- } \\
\text { xina }+ \text { hidroclorotiazida }\end{array}$ \\
\hline
\end{tabular}

Tabela 2-Manifestaçŏes clinicas em 19 pacientes com parkinsonismo indu:ido pela flunarizina.

\section{REFERENCIAS}

1. Bamrah JS, Soni SD - Drug-induced Parkinson's disease. Lancet 2:1031, 1987.

2. Borges M, Ghoos E, Thone F, Van Nueten JM - Effects of tlunarizine on the distribution of calcium in vascular smooth muscle. Blood Vessels 17:123, 1980.

3. Calne DB, Langston JW - Aetiology of Parkinson's disease. Lancet 2:1457, 1983.

4. Chouza C, Caamaño JC, Aljanati R, Scaramelli A, DeMedina O, Romero S $\longrightarrow$ Parkinsonism, tardive dyskinesia, akathisia, and depression induced by flunarizine. Lancet 1:1303, 1986.

5. Holmes B, Brogden RN, Heel RC, Speight TM, Avery GS - Flunarizine: a review of its pharmacodynamic and pharmacokinetic properties and therapeutic use. Drug 27:6, 1984.

6. Indo $\mathrm{T}$, Ando $\mathrm{K}-$ Metoclopramide-induced parkinsonism. Arch Neurol $39: 494,1982$.

7. Koller WC - Alcohol and Parkinson disease. Ann Neurol 13:466, 1983.

8. Micheli F, Pardal MF, Gatto M, Torres M, Paradiso MD, Pasesa IC, Giannaula MD Flunarizine and cinnarizine-induced extrapyramidul reaction. Neurology 37:881, 1987.

9. Sandyk R - Parkinsonism inducea by diazepan. Biol Psychiat 21:1229, 1986.

10. Stephen PJ, Williamson J - Drug-induced Parkinsonism in the elderly. Lancet 1:1082, 1984 .

11. Wilson JA, Primrose WR - Drug-induced parkinsonism. Br Med J 293:957, 1986.

12. Wilson JA, Primrose WR, Smith RG - Prognosis of drug-induced parkinsonism disease. Lancet 1:443, 1987. 